\title{
Integral Cohomology of 2-Local Hopf Spaces with at Most Two Non-Trivial Finite Homotopy Groups
}

\author{
Alain Clément \\ ABSTRACT. In this paper we prove that a non-contractible simply-connected \\ 2-local $H$-space $X$ with at most two non-trivial finite homotopy groups has no \\ homology exponent, i.e. no exponent $e \geqslant 1$ such that $e \cdot \widetilde{H}^{*}(X ; \mathbb{Z})=0$.
}

\section{Introduction}

Let $X$ be a connected topological space having the homotopy type of a CW-complex. One can both consider its graded homotopy group $\pi_{*}(X)$ and its graded reduced integral cohomology group $\widetilde{H}^{*}(X ; \mathbb{Z})$. If an integer $h \geqslant 1$ such that $h \cdot \pi_{*}(X)=0$ exists, then we say that $X$ has a homotopy exponent. Analogously, if an integer $e \geqslant 1$ such that $e \cdot \widetilde{H}^{*}(X ; \mathbb{Z})=0$ exists, then we say that $X$ admits a homology exponent.

A general question asked by D. Arlettaz suggests to explore the relationships between homotopy exponents and homology exponents. For instance, is it true that a space with a homotopy exponent has a homology exponent, too? In this case, how are these two exponents related? Or conversely, is it possible for a space without a homotopy exponent to admit a homology exponent?

In this paper, we focus on simply-connected 2-local $H$-spaces with one or two non-trivial finite homotopy groups. Such a space obviously admits a homotopy exponent. Our main result is the following:

MAIN THEOREM. Let $X$ be a non-contractible simply-connected 2-local H-space with at most two non-trivial finite homotopy groups. Then $X$ has no homology exponent.

The result when $X$ is an Eilenberg-Mac Lane space is a well-known consequence of the calculations of H. Cartan [2], see Corollary 2.5. More elaborated techniques are required to prove the result when $X$ has two non-trivial homotopy groups. Section 2 establishes some preparations and investigates the situation of EilenbergMac Lane spaces in detail. Some interesting examples are completely carried out in Section 3 and a proof of the main theorem is given in Section 4 . We conclude the paper with some questions and comments in Section 5.

2000 Mathematics Subject Classification. Primary 57T25; Secondary 55P20, 55S45.

Key words and phrases. Homology of $H$-spaces; Postnikov systems, $k$-invariants. 
Unless otherwise specified, a space will mean a pointed, connected and simple topological space with the homotopy type of a CW-complex of finite type. We will denote by $K(G, n)$ the Eilenberg-Mac Lane space with a single non-trivial homotopy group isomorphic to $G$ in dimension $n$ ( $G$ abelian if $n \geqslant 2)$.

Since we will only consider simple spaces $X$, we will only deal with abelian fundamental groups and it will always be possible to consider the Postnikov tower built up from the Postnikov sections of $X$ which will be denoted by $\alpha_{n}: X \longrightarrow X[n]$ and the $k$-invariants $k^{n+1}(X) \in H^{n+1}\left(X[n-1] ; \pi_{n} X\right) \cong$ $\left[X[n-1], K\left(\pi_{n} X, n+1\right)\right]$.

Acknowledgements. I would like to thank my thesis advisor D. Arlettaz for the help that he provided to me all along my studies and for the suggestions that he brought to this paper. I am also indebted to J. Scherer and C. Casacuberta for their support during my thesis work. I am very grateful to K. Hess-Belwald for her support and finally I would like to thank the referee who read this paper.

\section{Transverse implications}

In this introductory section we collect some well-known results that we need later in the paper, in particular results regarding the cohomology of 2-local EilenbergMac Lane spaces.

A non-empty finite sequence of positive integers $I=\left(a_{0}, \ldots, a_{k}\right)$, where $k$ is varying, is admissible if $a_{i} \geqslant 2 a_{i+1}$ for all $0 \leqslant i \leqslant k-1$. Let $\mathcal{S}$ be the set of all such admissible sequences. The stable degree is a map $\operatorname{deg}_{\text {st }}: \mathcal{S} \rightarrow \mathbb{N}$ defined by $\operatorname{deg}_{\text {st }}(I)=\sum_{i=0}^{k} a_{i}$ for all $I=\left(a_{0}, \ldots, a_{k}\right) \in \mathcal{S}$. The stable degree induces a grading on the set $\mathcal{S}$ of all admissible sequences. The excess is a map $e: \mathcal{S} \rightarrow \mathbb{N}$ defined by $e(I)=2 a_{0}-\operatorname{deg}_{\text {st }}(I)=a_{0}-\sum_{i=1}^{k} a_{i}$ for all $I=\left(a_{0}, \ldots, a_{k}\right) \in \mathcal{S}$.

Let $n \geqslant 1$ and $s \geqslant 1$. Let $\delta_{s}$ the connecting homomorphism in the long exact sequence of coefficients in cohomology associated to the short exact sequence

$$
0 \longrightarrow \mathbb{Z} / 2 \longrightarrow \mathbb{Z} / 2^{s+1} \longrightarrow \mathbb{Z} / 2^{s} \longrightarrow 0 \text {. }
$$

Consider the fundamental class $\iota_{n} \in H^{n}\left(K\left(\mathbb{Z} / 2^{s}, n\right) ; \mathbb{Z} / 2^{s}\right)$ and its mod-2 reduction $u_{n} \in H^{n}\left(K\left(\mathbb{Z} / 2^{s}, n\right) ; \mathbb{F}_{2}\right)$.

Convention. Let $I=\left(a_{0}, \ldots, a_{k}\right)$ be an admissible sequence. We will write $S q_{s}^{I} u_{n}$ instead of $S q^{a_{0}} \ldots S q^{a_{k-1}} \delta_{s} \iota_{n}$ (usually denoted by $S q^{a_{0}, \ldots, a_{k-1}} \delta_{s} \iota_{n}$ ) if $a_{k}=1$ and instead of $S q^{a_{0}} \ldots S q^{a_{k}} u_{n}$ (also denoted by $S q^{a_{0}, \ldots, a_{k}} u_{n}$ or $S q^{I} u_{n}$ ) if $a_{k} \neq 1$. In particular, since $\delta_{1}=S q^{1}$ and the reduction is the identity when $s=1$, we have $S q_{1}^{I} u_{n}=S q^{I} u_{n}$.

J.-P. Serre [11] computed the mod-2 cohomology of Eilenberg-Mac Lane spaces and stated the following result:

THEOREM 2.1. Let $n \geqslant 1$ and $s \geqslant 1$. The graded $\mathbb{F}_{2}$-algebra $H^{*}\left(K\left(\mathbb{Z} / 2^{s}, n\right) ; \mathbb{F}_{2}\right)$ is isomorphic to the graded polynomial $\mathbb{F}_{2}$-algebra on generators $S q_{s}^{I} u_{n}$, where $I$ covers all the admissible sequences of excess $e(I)<n$ and $u_{n}$ is the reduction of the fundamental class (see the above convention). The degree of a generator $S q_{s}^{I} u_{n}$ is $\operatorname{deg}\left(S q_{s}^{I} u_{n}\right)=\operatorname{deg}_{\text {st }}(I)+n$.

This result also reveals the $\mathcal{A}_{2}$-algebra structure of $H^{*}\left(K\left(\mathbb{Z} / 2^{s}, n\right) ; \mathbb{F}_{2}\right)$, where $\mathcal{A}_{2}$ denotes the mod-2 Steenrod algebra. 
It is well known that an Eilenberg-Mac Lane space associated with an abelian group has a unique $H$-space structure up to homotopy (which can be seen as inherited from the loop space structure or from the addition law of the associated abelian group). Therefore, the differential graded $\mathcal{A}_{2}$-algebra $H^{*}\left(K\left(\mathbb{Z} / 2^{s}, n\right) ; \mathbb{F}_{2}\right)$ is also a differential graded Hopf algebra. If $H$ is a graded Hopf algebra over the field $\mathbb{F}_{2}$, with multiplication $\mu: H \otimes H \rightarrow H$, comultiplication $\Delta: H \rightarrow H \otimes H$, augmentation $\epsilon: H \rightarrow \mathbb{F}_{2}$ and unit $\eta: \mathbb{F}_{2} \rightarrow H\left(\mathbb{F}_{2}\right.$ is concentrated in degree zero, see [9] for the definitions), the augmentation ideal of $H$ will be denoted by

$$
\bar{H}=\operatorname{ker} \epsilon: H \rightarrow \mathbb{F}_{2} \text {, }
$$

the graded module of indecomposable elements of $H$ by

$$
\begin{aligned}
Q H & =\bar{H} / \mu(\bar{H} \otimes \bar{H}) \\
& =\operatorname{coker} \mu: \bar{H} \rightarrow \bar{H} \otimes \bar{H}
\end{aligned}
$$

and the graded module of primitive elements of $H$ by

$$
\begin{aligned}
P H & =\{x \in \bar{H} \mid \Delta(x)=x \otimes 1+1 \otimes x\} \\
& =\operatorname{ker} \Delta: \bar{H} \rightarrow \bar{H} \otimes \bar{H} .
\end{aligned}
$$

The Milnor-Moore theorem states that there is an exact sequence of graded modules $0 \longrightarrow P(\xi H) \longrightarrow P H \longrightarrow Q H$, where $\xi H$ is the image of the Frobenius map $\xi: x \mapsto x^{2}$. The Hopf algebra $H$ is said to be primitively generated if $P H \rightarrow Q H$ in the above exact sequence is an epimorphism.

J.-P. Serre also proved the following key result in $[\mathbf{1 1}]$ :

THEOREM 2.2. The differential graded $\mathcal{A}_{2}$-algebra $H^{*}\left(K\left(\mathbb{Z} / 2^{s}, n\right) ; \mathbb{F}_{2}\right)$ is a connected, associative, commutative and primitively generated differential graded Hopf algebra for any integer $n \geqslant 1$ and any integer $s \geqslant 1$.

It is now easy to determine the modules of primitives and indecomposables of $H^{*}=H^{*}\left(K\left(\mathbb{Z} / 2^{s}, n\right) ; \mathbb{F}_{2}\right)$. The module of indecomposable elements is clearly given by

$$
Q H^{*} \cong \mathbb{F}_{2}\left\{S q_{s}^{I} u_{n} \mid I \text { admissible and } e(I)<n\right\} .
$$

Since $H^{*}$ is primitively generated, the Milnor-Moore theorem gives the following short exact sequence of graded $\mathbb{F}_{2}$-vector spaces:

$$
0 \longrightarrow P\left(\xi H^{*}\right) \longrightarrow P H^{*} \longrightarrow Q H^{*} \longrightarrow 0
$$

Therefore, every indecomposable element is primitive and every primitive element which is decomposable is a square of a primitive element. Thus we have

$$
P H^{*} \cong \mathbb{F}_{2}\left\{\left(S q_{s}^{I} u_{n}\right)^{2^{i}} \mid I \text { admissible, } e(I)<n \text { and } i \geqslant 0\right\} .
$$

Now let us present some concepts and results on the high torsion in the integral cohomology of Eilenberg-Mac Lane spaces associated with 2-torsion groups of finite type. The material exposed here can be found with all the details in my thesis work $[\mathbf{3}]$. It is mainly inspired by the work of H. Cartan in [2].

Let us start with the following key definition: 
Definition 2.3. Let $X$ be a space and $\left\{B_{r}^{*}, d_{r}\right\}$ be its mod-2 cohomology Bockstein spectral sequence $B_{1}^{*} \cong H^{*}\left(X ; \mathbb{F}_{2}\right) \Longrightarrow\left(H^{*}(X ; \mathbb{Z}) /\right.$ torsion $) \otimes \mathbb{F}_{2}$. Let $n$ and $r$ be two positive integers. An element $x \in B_{r}^{n}$ is said to be $\ell$-transverse if $d_{r+l} x^{2^{l}} \neq 0 \in B_{r+l}^{2^{l} n}$ for all $0 \leqslant l \leqslant \ell$. An element $x \in B_{r}^{n}$ is said to be $\infty$-transverse, or simply transverse, if it is $\ell$-transverse for all $\ell \geqslant 0$. We will also speak of transverse implications of an element $x \in B_{r}^{n}$.

For instance, suppose that $x \in B_{1}^{2}$ is $\infty$-transverse and let us picture how the transverse implications of $x$ look like within the Bockstein spectral sequence:

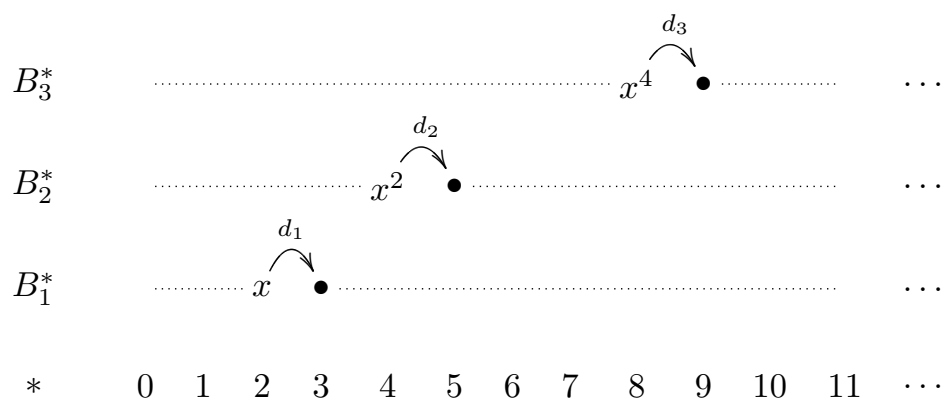

Every transverse element gives rise to 2-torsion of arbitrarily high order in the integral cohomology of $X$. Actually, our strategy for disproving the existence of a homology exponent for a space will consist in exhibiting a transverse element in its mod-2 cohomology Bockstein spectral sequence.

In the special case of Eilenberg-Mac Lane spaces, we have the following result:

Proposition 2.4. Let $G$ be a non-trivial 2-local abelian group of finite type isomorphic to $\mathbb{Z}_{(2)}^{\times s} \oplus \mathbb{Z} / 2^{s_{1}} \oplus \cdots \oplus \mathbb{Z} / 2^{s_{l}}$ and let $n \geqslant 2$. Consider the EilenbergMac Lane space $K(G, n)$ and its mod-2 cohomology Bockstein spectral sequence $\left\{B_{r}^{*}, d_{r}\right\}$. Suppose that one of the following assumptions holds:

- $n$ is even and $x \in B_{s_{j}}^{n}$ is 0 -transverse for any $1 \leqslant j \leqslant l$,

- $x \in P^{\text {even }} B_{1}^{*}$ is 0 -transverse $\left(S q^{1} x \neq 0\right)$.

Then $x$ is $\infty$-transverse.

A proof is given in [3, Theorem 1.3.2].

An algorithm explicitly computing the integral cohomology groups of such $K(G, n)$ spaces is implemented within a $\mathrm{C}++$ program in [4]: the Eilenberg-Mac Lane machine. For instance, the machine produces a table for $K(\mathbb{Z} / 2,2)$ whose part in low degrees is: 


\begin{tabular}{|l|l|l|}
\hline$n$ & $H^{n}(K(\mathbb{Z} / 2,2) ; \mathbb{Z})$ & $H^{n}\left(K(\mathbb{Z} / 2,2) ; \mathbb{F}_{2}\right)$ \\
\hline 0 & $\mathbb{Z}$ & $\mathbb{F}_{2}$ \\
1 & $(0)$ & $(0)$ \\
2 & $(0)$ & $\mathbb{F}_{2}\left\{u_{2}\right\}$ \\
3 & $\mathbb{Z} / 2$ & $\mathbb{F}_{2}\left\{S q^{1} u_{2}\right\}$ \\
4 & $(0)$ & $\mathbb{F}_{2}\left\{u_{2}^{2}\right\}$ \\
5 & $\mathbb{Z} / 2^{2}$ & $\mathbb{F}_{2}\left\{S q^{2,1} u_{2}, u_{2} S q^{1} u_{2}\right\}$ \\
6 & $\mathbb{Z} / 2$ & $\mathbb{F}_{2}\left\{u_{2}^{3},\left(S q^{1} u_{2}\right)^{2}\right\}$ \\
7 & $\mathbb{Z} / 2$ & $\mathbb{F}_{2}\left\{u_{2} S q^{2,1} u_{2}, u_{2}^{2} S q^{1} u_{2}\right\}$ \\
8 & $\mathbb{Z} / 2$ & $\mathbb{F}_{2}\left\{u_{2}^{4}, u_{2}\left(S q^{1} u_{2}\right)^{2}, S q^{1} u_{2} S q^{2,1} u_{2}\right\}$ \\
9 & $\mathbb{Z} / 2 \oplus \mathbb{Z} / 2^{3}$ & $\mathbb{F}_{2}\left\{S q^{4,2,1} u_{2}, u_{2}^{2} S q^{2,1} u_{2}, u_{2}^{3} S q^{1} u_{2},\left(S q^{1} u_{2}\right)^{3}\right\}$ \\
\hline
\end{tabular}

The elements of order 2, 4 and 8 in degrees 3,5 and 9 respectively are given by an $\infty$-transverse element: the characteristic class $u_{2} \in H^{2}\left(K(\mathbb{Z} / 2,2) ; \mathbb{F}_{2}\right)$ - which is of even degree and 0 -transverse - and its iterated squares $u_{2}^{2}$ and $u_{2}^{4}$.

Let us remark that a 0 -transverse implication does not imply $\infty$-transverse implications in general. More precisely, the fact that $x \in P^{\text {even }} H^{*}\left(X ; \mathbb{F}_{2}\right)$ is such that $S q^{1} x \neq 0$ does not always force $x$ to be $\infty$-transverse. A counter-example is given by $X=B S O$ and $x=w_{2}$, the second Stiefel-Withney class in $H^{2}\left(B S O ; \mathbb{F}_{2}\right)$.

As a corollary of Proposition 2.4, it is then possible to give a proof of our main result for Eilenberg-Mac Lane spaces:

COROLlary 2.5. Let $G$ be a non-trivial finite 2-torsion abelian group and let $n \geqslant 2$. The Eilenberg-Mac Lane space $K(G, n)$ has no homology exponent.

Proof. Accordingly to the Künneth formula, it is sufficient to establish the result when $G=\mathbb{Z} / 2^{s}$ for some $s \geqslant 1$. If $n$ is even, consider the reduction of the fundamental class $u_{n} \in H^{n}\left(K\left(\mathbb{Z} / 2^{s}, n\right) ; \mathbb{F}_{2}\right)$. This class survives to $B_{s}^{n}$ and is 0 -transverse. Then $u_{n} \in B_{s}^{n}$ is $\infty$-transverse. If $n$ is odd, consider the admissible sequence $(2,1)$. Its excess is exactly 1 and therefore $S q_{s}^{2,1} u_{n} \in P^{\text {even }} H^{*}\left(K(\mathbb{Z} / 2, n) ; \mathbb{F}_{2}\right)$ when $n \geqslant 3$. Moreover we have $S q^{1} S q_{s}^{2,1} u_{n}=S q_{s}^{3,1} u_{n}$ by Adem relations, which means that $S q_{s}^{2,1} u_{n}$ is 0 -transverse. Hence $S q_{s}^{2,1} u_{n} \in B_{1}^{n+3}$ is $\infty$-transverse.

Let us conclude this section by proving the following crucial observation which states that the $\infty$-transverse implications of an element in the cohomology of the total space of a fibration can be read in the cohomology of the fibre.

LEMMA 2.6. Let $j: F \rightarrow X$ be a continuous map. If $x \in H^{*}\left(X ; \mathbb{F}_{2}\right)$ is such that $j^{*}(x) \neq 0 \in H^{*}\left(F ; \mathbb{F}_{2}\right)$ is $\infty$-transverse, then $x$ itself is $\infty$-transverse.

Proof. Suppose that $x$ is not $\infty$-transverse. Then there exists $r \geqslant 0$ such that $d_{r+1} x^{2^{r}}=0$. Therefore we have $d_{r+1} j^{*}(x)^{2^{r}}=d_{r+1} j^{*}\left(x^{2^{r}}\right)=j^{*} d_{r+1} x^{2^{r}}=0$, since $d_{r+1} x^{2^{r}}=0$, which contradicts $\infty$-transversity of $j^{*}(x)$.

\section{Examples}

Let us consider now 2-local spaces with two non-trivial finite homotopy groups. Recall that we want to prove that they do not have a homology exponent. This section will be devoted to two interesting examples. 
EXAMPLE 3.1. If such a space $X$ retracts onto an Eilenberg-Mac Lane space, then it is a easy to deduce that $X$ has no homology exponent. This is for instance the case for the space $X$ given by the fibration

$$
X \stackrel{i}{\longrightarrow} K(\mathbb{Z} / 2,2) \times K(\mathbb{Z} / 2,2) \stackrel{k}{\longrightarrow} K(\mathbb{Z} / 2,4),
$$

where its single non-trivial $k$-invariant

$$
\begin{aligned}
k \in & {[K(\mathbb{Z} / 2,2) \times K(\mathbb{Z} / 2,2), K(\mathbb{Z} / 2,4)] } \\
\cong & H^{4}\left(K(\mathbb{Z} / 2,2) \times K(\mathbb{Z} / 2,2) ; \mathbb{F}_{2}\right) \\
\cong & H^{4}\left(K(\mathbb{Z} / 2,2) ; \mathbb{F}_{2}\right) \otimes \mathbb{F}_{2} \\
& \oplus H^{2}\left(K(\mathbb{Z} / 2,2) ; \mathbb{F}_{2}\right) \otimes H^{2}\left(K(\mathbb{Z} / 2,2) ; \mathbb{F}_{2}\right) \\
& \oplus \mathbb{F}_{2} \otimes H^{4}\left(K(\mathbb{Z} / 2,2) ; \mathbb{F}_{2}\right) \\
\cong & \mathbb{F}_{2}\left\{u_{2}^{2} \otimes 1, u_{2} \otimes v_{2}, 1 \otimes v_{2}^{2}\right\}
\end{aligned}
$$

is given by $k=u_{2} \otimes v_{2}$ where $u_{2}$ and $v_{2}$ are the fundamental classes of both copies of $K(\mathbb{Z} / 2,2)$. The space $X$ has only two non-trivial homotopy groups $\pi_{2}(X) \cong$ $\mathbb{Z} / 2 \oplus \mathbb{Z} / 2$ and $\pi_{3}(X) \cong \mathbb{Z} / 2$.

Proposition 3.2. The space $X$ of Example 3.1 has the following properties:

1. $X$ is not a GEM (i.e. a weak product of Eilenberg-Mac Lane spaces),

2. $X$ is not a $H$-space,

3. $X$ retracts (weakly) onto the Eilenberg-Mac Lane space $K(\mathbb{Z} / 2,2)$, i.e. there exist maps $f: X \rightarrow K(\mathbb{Z} / 2,2)$ and $g: K(\mathbb{Z} / 2,2) \rightarrow X$ such that $f g \simeq \operatorname{id}_{K(\mathbb{Z} / 2,2)}$,

4. $f^{*}: H^{*}\left(K(\mathbb{Z} / 2,2) ; \mathbb{F}_{2}\right) \rightarrow H^{*}\left(X ; \mathbb{F}_{2}\right)$ is a monomorphism,

5. $X$ has no homology exponent.

Proof. The space $X$ is clearly not a GEM nor a $H$-space.

Consider the following homotopy commutative diagram based on the fibration for which $X$ is the fibre:

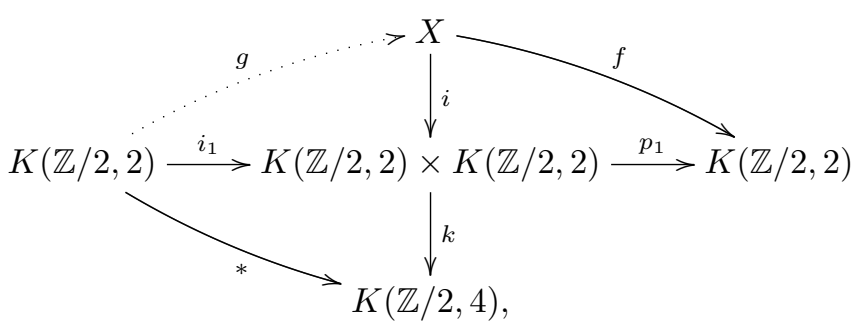

where $i_{1}$ denotes the inclusion into the first factor, $p_{1}$ denotes the projection onto the first factor and $f=p_{1} i$. The existence of a (generally not unique) map $g$ is a consequence of the fact that $k i_{1} \simeq *$. To see that $k i_{1} \simeq *$, recall first that the isomorphism $[K(\mathbb{Z} / 2,2), K(\mathbb{Z} / 2,4)] \cong H^{4}\left(K(\mathbb{Z} / 2,2) ; \mathbb{F}_{2}\right)$ maps $k i_{1}$ to $\left(k i_{1}\right)^{*}\left(u_{4}\right)$, where $\left(k i_{1}\right)^{*}=\left(i_{1}\right)^{*} k^{*}: H^{4}\left(K(\mathbb{Z} / 2,4) ; \mathbb{F}_{2}\right) \rightarrow H^{4}\left(K(\mathbb{Z} / 2,2) ; \mathbb{F}_{2}\right)$ and $u_{4} \in H^{4}\left(K(\mathbb{Z} / 2,4) ; \mathbb{F}_{2}\right)$ is the fundamental class. Now we have $\left(i_{1}\right)^{*} k^{*}\left(u_{4}\right)=$ $\left(i_{1}\right)^{*}\left(u_{2} \otimes v_{2}\right)=\left(i_{1}\right)^{*}\left(u_{2} \otimes 1 \cdot 1 \otimes v_{2}\right)=\left(i_{1}\right)^{*}\left(u_{2} \otimes 1\right) \cdot\left(i_{1}\right)^{*}\left(1 \otimes v_{2}\right)=0$, since $\left(i_{1}\right)^{*}\left(1 \otimes v_{2}\right)=0$. Therefore $f g \simeq$ id and the result follows. 
One can relate this space with other examples pointed out by F. R. Cohen and F. P. Peterson in [5]. They constructed loop maps $\Omega g: \Omega Y \rightarrow K(\mathbb{Z} / 2, n)$, $n \geqslant 2$, with the property that $(\Omega g)^{*}: H^{*}\left(K(\mathbb{Z} / 2, n) ; \mathbb{F}_{2}\right) \rightarrow H^{*}\left(\Omega Y ; \mathbb{F}_{2}\right)$ is a monomorphism and such that $\Omega g$ does not admit a section.

Their examples are mainly provided by $\Omega \Sigma\left(\mathbb{R} P^{\infty}\right)^{n} \rightarrow K(\mathbb{Z} / 2, n)$, the canonical multiplicative extension of Serre's map $e:\left(\mathbb{R} P^{\infty}\right)^{n} \rightarrow K(\mathbb{Z} / 2, n)$, and by $\Omega \Sigma B S O(3) \rightarrow K(\mathbb{Z} / 2,2)$, the canonical multiplicative extension of the second Stiefel-Whitney class in the mod-2 cohomology of $B S O(3)$ in the case $n=2$.

The spaces $\Sigma\left(\mathbb{R} P^{\infty}\right)^{n}$ and $\Sigma B S O(3)$ have infinitely many non-trivial homotopy groups. Our space $X$ has only two. However, the loop maps $\Omega g: \Omega Y \rightarrow K(\mathbb{Z} / 2, n)$ of F. R. Cohen and F. P. Peterson and our map $f: X \rightarrow K(\mathbb{Z} / 2,2)$ all induce monomorphisms.

EXAMPLE 3.3. Let us now consider a more interesting example of 2-local space with two non-trivial homotopy groups which does not admit a retract onto an Eilenberg-Mac Lane space. Therefore we will not be able to use the topological argument of the proof of Proposition 3.2 in order to prove the non-existence of a homology exponent. The main idea here is to detect $\infty$-transverse implications.

Let $X$ be the space given by the fibration

$$
X \stackrel{i}{\longrightarrow} K(\mathbb{Z} / 2,2) \stackrel{k}{\longrightarrow} K(\mathbb{Z} / 2,4)
$$

where its single non-trivial $k$-invariant

$$
\begin{aligned}
k & \in[K(\mathbb{Z} / 2,2), K(\mathbb{Z} / 2,4)] \\
& \cong H^{4}\left(K(\mathbb{Z} / 2,2) ; \mathbb{F}_{2}\right) \\
& \cong \mathbb{F}_{2}\left\{u_{2}^{2}\right\}
\end{aligned}
$$

is given by $k=u_{2}^{2}$ where $u_{2}$ is the fundamental class of $K(\mathbb{Z} / 2,2)$. The space $X$ has only two non-trivial homotopy groups $\pi_{2}(X) \cong \mathbb{Z} / 2 \cong \pi_{3}(X)$.

Proposition 3.4. The space $X$ of Example 3.3 has the following properties:

1. $X$ is not a GEM,

2. $X$ is an infinite loop space,

3. $X$ retracts neither onto the Eilenberg-Mac Lane space $K(\mathbb{Z} / 2,2)$, nor onto $K(\mathbb{Z} / 2,3)$

4. However, $X$ has no homology exponent.

Proof. The space $X$ is clearly not a GEM. It is an infinite loop space since its $k$-invariant $u_{2}^{2}$ is in the image of the $n$-fold cohomology suspension for all $n$.

In order to show that $X$ does retract neither onto $K(\mathbb{Z} / 2,2)$, nor onto $K(\mathbb{Z} / 2,3)$, let us consider the mod-2 cohomology Serre spectral sequence of the fibration 
$K(\mathbb{Z} / 2,3) \stackrel{j}{\longrightarrow} X \stackrel{i}{\longrightarrow} K(\mathbb{Z} / 2,2)$. The $E_{2}$-term looks like:

\begin{tabular}{c|cccccc}
$S q^{1} u_{3}$ & 0 & $*$ & $*$ & $*$ & $*$ & $*$ \\
$u_{3}$ & 0 & $u_{2} u_{3}$ & $*$ & $*$ & $*$ & $*$ \\
0 & 0 & 0 & 0 & 0 & 0 & 0 \\
0 & 0 & 0 & 0 & 0 & 0 & 0 \\
\hline 1 & 0 & $u_{2}$ & $S q^{1} u_{2}$ & $u_{2}^{2}$ & $S q^{2,1} u_{2}$ & $u_{2}^{3}$ \\
& & & & & $u_{2} S q^{1} u_{2}$ & $\left(S q^{1} u_{2}\right)^{2}$
\end{tabular}

We have $H^{2}\left(X ; \mathbb{F}_{2}\right) \cong \mathbb{F}_{2}\{v\}$ with $u_{2} \mapsto v$ via the composition

$$
i^{*}: H^{2}\left(K(\mathbb{Z} / 2,2) ; \mathbb{F}_{2}\right) \cong E_{2}^{2,0} \longrightarrow E_{3}^{2,0} \cong E_{\infty}^{2,0} \cong H^{2}\left(X ; \mathbb{F}_{2}\right)
$$

The Serre's transgression theorem (see for instance [8, Theorem 6.8, p. 189]) implies that $d_{4}$ coincides with the transgression which is given by the $k$-invariant. In other words we have $d_{4} u_{3}=k^{*}\left(u_{4}\right)=u_{2}^{2}$. Therefore $\bigoplus_{s} E_{\infty}^{s, 3-s} \cong E_{\infty}^{3,0}$ and $H^{3}\left(X ; \mathbb{F}_{2}\right) \cong \mathbb{F}_{2}\{w\}$ with $S q^{1} u_{2} \mapsto w$ via the composition

$$
i^{*}: H^{*}\left(K(\mathbb{Z} / 2,2) ; \mathbb{F}_{2}\right) \cong E_{2}^{3,0} \longrightarrow E_{3}^{3,0} \longrightarrow E_{4}^{3,0} \cong E_{\infty}^{3,0} \cong H^{3}\left(X ; \mathbb{F}_{2}\right) .
$$

We clearly have $S q^{1} v=w$.

Suppose that there are maps $f: X \rightarrow K(\mathbb{Z} / 2,2)$ and $g: K(\mathbb{Z} / 2,2) \rightarrow X$ with $f g \simeq \operatorname{id}_{K(\mathbb{Z} / 2,2)}$. The only non-trivial map $f: X \rightarrow K(\mathbb{Z} / 2,2)$ is given by the single non-trivial element $v \in H^{2}\left(X ; \mathbb{F}_{2}\right)$. This forces $f \simeq i$. Therefore we have $k i g \simeq k \operatorname{id}_{K(\mathbb{Z} / 2,2)} \simeq k$ which contradicts the fact that $k i \simeq *$.

Suppose now that there are maps $f: X \rightarrow K(\mathbb{Z} / 2,3)$ and $g: K(\mathbb{Z} / 2,3) \rightarrow X$ with $f g \simeq \operatorname{id}_{K(\mathbb{Z} / 2,3)}$. The only non-trivial map $f: X \rightarrow K(\mathbb{Z} / 2,3)$ is given by the single non-trivial element $w \in H^{3}\left(X ; \mathbb{F}_{2}\right)$. Therefore we have $g^{*} f^{*}\left(u_{3}\right)=g^{*}(w)=$ $g^{*}\left(S q^{1} v\right)=S q^{1} g^{*}(v)=0$, since $g^{*}(v) \in H^{2}\left(K(\mathbb{Z} / 2,3) ; \mathbb{F}_{2}\right)=0$. In other words, we always have $f g \simeq *$ and $X$ cannot retract onto $K(\mathbb{Z} / 2,3)$.

Since $u_{3}$ transgresses to $u_{2}^{2}, S q^{1} u_{3}$ transgresses to $S q^{1} u_{2}^{2}$ which vanishes by Cartan's formula. Therefore $S q^{1} u_{3} \neq 0 \in E_{\infty}^{0,4}$ and there exists $x^{\prime} \in H^{4}\left(X ; \mathbb{F}_{2}\right)$ such that $x^{\prime} \mapsto S q^{1} u_{3}$ via the composition

$$
j^{*}: H^{4}\left(X ; \mathbb{F}_{2}\right) \longrightarrow E_{\infty}^{0,4} \cong E_{6}^{0,4} \subset \cdots \subset E_{2}^{0,4} \cong H^{4}\left(K(\mathbb{Z} / 2,3) ; \mathbb{F}_{2}\right) .
$$

Set $x=S q^{2} x^{\prime}$. We have $j^{*}(x)=j^{*}\left(S q^{2} x^{\prime}\right)=S q^{2} j^{*}\left(x^{\prime}\right)=S q^{2,1} u_{3}$ which is $\infty$ transverse. Thus $x$ is also $\infty$-transverse and $X$ cannot admit a homology exponent.

\section{Proof of the main result}

This section is devoted to the proof of our main result. We begin to sketch a strategy allowing us to reach our aim. 
Strategy. In Example 3.3, we found an element $x$ in the mod-2 cohomology of $X$ such that its image in the cohomology of the fibre $j^{*}(x)=S q^{2,1} u_{3}$ is non-trivial and has $\infty$-transverse implications; this imply for $x$ itself to have $\infty$-transverse implications and prevents the existence of a homology exponent. Let us consider each of these stages in the general setting of a space $X$ with two non-trivial homotopy groups:

(A) Every non-trivial element in $E_{2}^{0, *}$ which survives to $E_{\infty}^{0, *}$ gives rise to an element $x \in H^{*}\left(X ; \mathbb{F}_{2}\right)$ with a non-trivial image $j^{*}(x)$ in the cohomology of the fibre. This leads us to find an element in $E_{2}^{0, *}$ which transgresses to zero. The fact that transgressions in the spectral sequence commute with the Steenrod squares is very valuable for our purpose (J.-P. Serre remarked that the transgressions also commute with the connecting homomorphism $\delta_{s}$ introduced in Section 2, see [11, p. 206] and [10, p. 457]). For instance, the reduction of the characteristic class $u_{n} \in E_{2}^{0, n}$ transgresses to a primitive element $w$ determined by the $k$-invariant. Therefore, the first step will be done if we can find an admissible sequence $I$ such that $S q_{s}^{I} u_{n} \neq 0$ transgresses to $S q_{s}^{I} w=0$.

(B) Among all the admissible sequences $I=\left(a_{0}, \ldots\right)$ of excess $e(I)<n$ such that $S q_{s}^{I} u_{n} \neq 0$ transgresses to zero, some of them are interesting because they insure on one hand that the element $S q_{s}^{I} u_{n}$ has a 0 -transverse implication in the cohomology of the fibre and on the other hand that $S q_{s}^{I} u_{n}$ lies in even degree. We have seen in Proposition 2.4 that these two conditions force $S q_{s}^{I} u_{n}$ to have $\infty$-implications. It is immediate to see that such an admissible sequence "begins" with an even $a_{0}$ and has stable degree $\operatorname{deg}_{\mathrm{st}}(I) \equiv n(\bmod 2)$.

Let us look at the $\mathcal{A}_{2}$-action on the primitive elements of the cohomology of the base space. Following (A), our aim here is to find a suitable admissible sequence. Consider the following definition:

Definition 4.1. For all $l \geqslant 1$ define the admissible sequence

$$
\xi(l)=\left(2^{l}+2^{l-1}-1, \ldots, 5,2\right)
$$

of stable degree $\operatorname{deg}_{\text {st }}(\xi(l))=2^{l+1}+2^{l}-l-3$ and excess $e(\xi(l))=l+1$.

Proposition 4.2. Let $m \geqslant 2$ and let $G$ be a 2 -torsion finite abelian group and consider the $\mathcal{A}_{2}$-module of primitives $P^{*} H^{*}\left(K(G, m) ; \mathbb{F}_{2}\right)$. Let $n \geqslant m+1$. Then we have

$$
\begin{cases}S q^{2} P^{5} H^{*}\left(K(G, 2) ; \mathbb{F}_{2}\right)=0 . & \\ S q^{3} P^{6} H^{*}\left(K(G, 3) ; \mathbb{F}_{2}\right)=0 . & \\ S q^{3} P^{n+1} H^{*}\left(K(G, m) ; \mathbb{F}_{2}\right)=0 & \text { if } n=m+1 \geqslant 4 . \\ S q^{\xi(n-3)} P^{n+1}\left(K(G, m) ; \mathbb{F}_{2}\right)=0 & \text { if } n \geqslant m+2 .\end{cases}
$$

Proof. Since $G$ is a 2 -torsion finite abelian group, we can write $G \cong \oplus_{a} \mathbb{Z} / 2^{s_{a}}$.

Suppose that $n=m+1=3$. Let $i_{2} \in H^{2}(K(G, 2) ; G)$ be the characteristic class and consider the elements $\iota_{2, a} \in H^{2}\left(K(G, 2) ; \mathbb{Z} / 2^{s_{a}}\right)$ induced by the projections of $G$ on each of the factors $\mathbb{Z} / 2^{s_{a}}$. Let $x \in P^{5} H^{*}\left(K(G, 2) ; \mathbb{F}_{2}\right)$. There exists an element $y \in H^{3}\left(K(G, 2) ; \mathbb{F}_{2}\right)$ of the form $y=\sum \delta_{s} \iota_{2, s}$ and such that $x=S q^{2} y$. We have $S q^{2} x=S q^{2} S q^{2} y=S q^{3,1} y=\sum S q^{3,1} \delta_{s} \iota_{2, s}=0$ since $S q^{1} \delta_{s}=0$ for all $s$. 
Suppose that $n=m+1=4$ and let $x \in P^{6} H^{*}\left(K(G, 3) ; \mathbb{F}_{2}\right)$. There exists $y \in H^{3}\left(K(G, 3) ; \mathbb{F}_{2}\right)$ such that $x=y^{2}$. We then have $S q^{3} x=S q^{3} y^{2}=0$ by Cartan's formula.

Suppose that $n=m+1 \geqslant 4$ and let $x \in P^{m+2} H^{*}\left(K(G, m) ; \mathbb{F}_{2}\right)$. There exists $y \in H^{m}\left(K(G, m) ; \mathbb{F}_{2}\right)$ such that $x=S q^{2} y$. We then have $S q^{3} x=S q^{3} S q^{2} y=0$ by Adem relations.

Finally suppose that $n \geqslant m+2$. For all $m \geqslant 2$ define the following subsets of the integers:

$$
\begin{aligned}
M^{m} & =\left\{2^{i}+2^{i-1} \mid \text { for all } i \geqslant m\right\} \text { and } \\
N^{m} & =\left\{1+2^{h_{1}}+\cdots+2^{h_{m-1}} \mid h_{1} \geqslant \ldots \geqslant h_{m-1} \geqslant 0\right\} .
\end{aligned}
$$

It is a very simple arithmetic game to see that $M^{m} \cap N^{m}=\emptyset$. Careful calculations show that for all admissible sequence $I$, we have $e(I)<m$ if and only if $\operatorname{deg}_{\text {st }}(I)+$ $m \in N^{m}$. Thus there is no admissible sequence $I$ of excess $e(I)<m$ such that $\operatorname{deg}_{\text {st }}(I)+m=2^{\geqslant m}+2^{\geqslant m-1}$. Therefore

$$
Q^{2^{\geqslant m}+2^{\geqslant m-1}} H^{*}\left(K(G, m) ; \mathbb{F}_{2}\right)=0 .
$$

Let $x \in P^{n+1} H^{*}\left(K(G, m) ; \mathbb{F}_{2}\right)$. We have

$$
\begin{aligned}
\operatorname{deg}\left(S q^{2^{n-3}+2^{n-4}-2} S q^{\xi(n-4)} x\right) & =\operatorname{deg}(x)+\operatorname{deg}_{s t}(\xi(n-4))+\left(2^{n-3}+2^{n-4}-2\right) \\
& =2^{n-2}+2^{n-3}
\end{aligned}
$$

Since $Q^{2^{n-2}+2^{n-3}} H^{*}\left(K(G, m) ; \mathbb{F}_{2}\right)=0$ when $n \geqslant m+2$, the primitive element $S q^{2^{n-3}+2^{n-4}-2} S q^{\xi(n-4)} x$ is then decomposable. Thus it is a square (maybe trivial) by Milnor-Moore. Therefore

$$
\begin{aligned}
S q^{\xi(n-3)} x & =S q^{2^{n-3}+2^{n-4}-1} S q^{\xi(n-4)} x \\
& =S q^{1} S q^{2^{n-3}+2^{n-4}-2} S q^{\xi(n-4)} x \\
& =S q^{1} \text { (square) }=0 .
\end{aligned}
$$

We are now able to prove the main theorem.

THEOREM 4.3. Let $X$ be a non-contractible simply-connected 2-local H-space with at most two non-trivial finite homotopy groups. Then $X$ has no homology exponent.

Proof. Since the case of an Eilenberg-Mac Lane space is clear, let us assume that $X$ is a non-contractible simply-connected 2-local $H$-space with exactly two non-trivial homotopy groups $\pi_{m}(X) \cong G$ and $\pi_{n}(X) \cong H$, where $n>m \geqslant 2$ and $G \cong \oplus_{a} \mathbb{Z} / 2^{s_{a}}, H \cong \oplus_{b} \mathbb{Z} / 2^{t_{b}}$ are finite groups.

The space $X$ fits into the fibrations

$$
K(H, n) \stackrel{j}{\longrightarrow} X \stackrel{i}{\longrightarrow} K(G, m) \stackrel{k}{\longrightarrow} K(H, n+1),
$$

where $k$ is its single $k$-invariant. Since $X$ is a $H$-space, $k$ is a $H$-map.

Let $i_{m} \in H^{2}(K(G, m) ; G)$ be the characteristic class and consider the elements $\iota_{m, a} \in H^{m}\left(K(G, m) ; \mathbb{Z} / 2^{s_{a}}\right)$ induced by the projections of $G$ on each factor $\mathbb{Z} / 2^{s_{a}}$. Consider also all the $u_{m, a} \in H^{m}\left(K(G, m) ; \mathbb{F}_{2}\right)$ given by the reduction mod 2 of the $\iota_{m, a}$ 's. 
Analogously, let $j_{n} \in H^{n}(K(H, n) ; H)$ be the characteristic class and consider the elements $\jmath_{n, b} \in H^{n}\left(K(H, n) ; \mathbb{Z} / 2^{t_{b}}\right)$ induced by the projections of $H$ on each factor $\mathbb{Z} / 2^{t_{b}}$. Consider also all the $v_{n, b} \in H^{n}\left(K(H, n) ; \mathbb{F}_{2}\right)$ given by the reduction $\bmod 2$ of the $\jmath_{n, b}$ 's. Moreover, pick $\left(t, v_{n}, \jmath_{n}\right)$ among $\left\{\left(t_{b}, v_{n, b}, \jmath_{n, b}\right) \mid\right.$ for all $\left.b\right\}$.

The $E_{2}$-term of the Serre spectral sequence of the fibration

$$
K(H, n) \longrightarrow X \longrightarrow K(G, m)
$$

looks like:

$$
\begin{array}{c|cccccc}
\delta_{t} J_{n}, * & 0 & \cdots & 0 & * & * & * \\
v_{n}, * & 0 & \cdots & 0 & * & * & * \\
0 & 0 & \cdots & 0 & 0 & 0 & 0 \\
\vdots & \vdots & & \vdots & \vdots & \vdots & \vdots \\
0 & 0 & \cdots & 0 & 0 & 0 & 0 \\
\hline 1 & 0 & \cdots & 0 & * & * & *
\end{array}
$$

The element $v_{n}$ transgresses to $d_{n+1} v_{n}$ in $P^{n+1} H^{*}\left(K(G, m) ; \mathbb{F}_{2}\right)$ which is determined by the $k$-invariant. Set

$$
\xi= \begin{cases}(2,1) & \text { if } m=2 \text { and } n=3, \\ (6,3,1) & \text { if } m=3 \text { and } n=4, \\ (6,3) & \text { if } n=m+1 \geqslant 5 \text { and } n \text { is odd, } \\ (14,7,3) & \text { if } n=m+1 \geqslant 6 \text { and } n \text { is even, } \\ \left(2^{n-2}+2^{n-3}-2, \xi(n-3)\right) & \text { if } n \geqslant m+2 \geqslant 4 .\end{cases}
$$

In all cases $e(\xi)<n, \operatorname{deg}\left(S q_{t}^{\xi} v_{n}\right)$ is even and $S q_{t}^{\xi} v_{n}$ transgresses to $S q_{t}^{\xi} d_{n+1} v_{n}$ which is trivial by Proposition 4.2. Let $x \in H^{*}\left(X ; \mathbb{F}_{2}\right)$ such that $j^{*}(x)=S q_{t}^{\xi} v_{n}$. The element $S q_{t}^{\xi} v_{n}$ is $\infty$-transverse and so is $x$.

\section{Generalizations}

We conclude this paper with some possible generalizations of our main result.

The first generalization in which we are interested concerns the nature of the two non-trivial homotopy groups of $X$. We supposed them to be finite. Let us now suppose that the homotopy groups of $X$ are of finite type. Copies of $\mathbb{Z}_{(2)}$, which have no torsion, may appear in this extended context.

The cohomology of Eilenberg-Mac Lane spaces associated to such groups was also computed by H. Cartan and J.-P. Serre:

$$
H^{*}\left(K\left(\mathbb{Z}_{(2)}, n\right) ; \mathbb{F}_{2}\right) \cong \mathbb{F}_{2}\left[S q^{I} u_{n} \mid I=\left(a_{0}, \ldots, a_{k}\right) \text { with } a_{k} \neq 1 \text { and } e(I)<n\right] .
$$

We say that a space $X$ admits a torsion homology exponent if there exists an exponent for the torsion subgroup of $H^{*}(X ; \mathbb{Z})$. With this definition, we have the following result on Eilenberg-Mac Lane spaces:

Proposition 5.1. Let $G$ be a non-trivial 2-torsion abelian group of finite type and $n \geqslant 4$. The Eilenberg-Mac Lane space $K(G, n)$ has no torsion homology exponent. 
Proof. By Künneth formula and Corollary 2.5, it is sufficient to suppose $G=$ $\mathbb{Z}_{(2)}$. Consider the reduction of the fundamental class $u_{n} \in H^{n}\left(K\left(\mathbb{Z}_{(2)}, n\right) ; \mathbb{F}_{2}\right)$. If $n$ is even, then $S q^{2} u_{n}$ is $\infty$-transverse. If $n$ is odd, then $S q^{6,3} u_{n}$ is $\infty$-transverse.

It is not very difficult using results of $\mathrm{H}$. Cartan in [2] to verify that $K\left(\mathbb{Z}_{(2)}, 2\right)$ and $K\left(\mathbb{Z}_{(2)}, 3\right)$ admit torsion homology exponents. So the result is the best possible in terms of connexity. The following result is an extension to spaces with at most two non-trivial homotopy groups of finite type:

TheOREM 5.2. Let $X$ be a non-contractible 3-connected 2-local $H$-space of finite type with at most two non-trivial homotopy groups. Then $X$ has no torsion homology exponent.

The strategy and the admissible sequences $\xi$ listed in the proof of Theorem 4.3 are also suitable to prove Theorem 5.2.

W. Browder proved in $[\mathbf{1}$, Theorem 6.11, p. 46] that every $H$-space of finite type which has the homotopy type of a finite $\mathrm{CW}$-complex and which is simplyconnected is actually 2 -connected. Then, one may ask the following question.

Question 5.3. Let $X$ be a simply-connected 2-local $H$-space of finite type with a homology exponent. Is $X$ always 2-connected? If it is not the case for all such $H$-spaces, is it true for infinite loop spaces?

In [7] R. Levi studied the homotopy type of $p$-completed classifying spaces of the form $B G_{p}^{\wedge}$ for $G$ a finite $p$-perfect group, $p$ a prime. He constructed an algebraic analogue of Quillen's "plus" construction for differential graded coalgebras. He then proved that the loop spaces $\Omega B G_{p}^{\wedge}$ admit integral homology exponents. More precisely, he proved that if $G$ is a finite $p$-perfect group of order $p^{r} \cdot m, m$ prime to $p$, then

$$
p^{r} \cdot \widetilde{H}_{*}\left(\Omega B G_{p}^{\wedge} ; \mathbb{Z}_{(p)}\right)=0 .
$$

Moreover, he proved that $B G_{p}^{\wedge}$ admits in general infinitely many non-trivial $k$ invariants, and thus in particular $\pi_{*} B G_{p}^{\wedge}$ is non-trivial in arbitrarily high dimensions. His method for proving this last result is based on a version of H. Miller's theorem improved by J. Lannes and L. Schwartz [6]. This result and the results of the paper lead to the following conjecture:

ConjeCture 5.4. Let $X$ be a connected space. If $X$ has a homology exponent, then either $X \simeq K\left(\pi_{1}(X), 1\right)$, or $X$ has infinitely many non-trivial $k$-invariants and, in particular, infinitely many non-trivial homotopy groups.

One can attack this conjecture by first looking at the following problem at the prime 2:

QUESTION 5.5. Let $X$ be a 2-local space (of finite type) and $G$ a finite 2-torsion abelian group. If $X$ has a homology exponent, is the $\operatorname{space}^{\text {map }}$ * $(K(G, 2), X)$ weakly contractible?

To see that an affirmative answer to this question implies Conjecture 5.4 at the prime 2, suppose that $X$ is a 2-local space with a homology exponent and with finitely many non-trivial $k$-invariants. Then $X \simeq X[m] \times$ GEM for some integer $m$. 
Since $X$ admits a homology exponent it is a Postnikov piece. Consider then the Postnikov tower of the space $X \simeq X[m]$ :

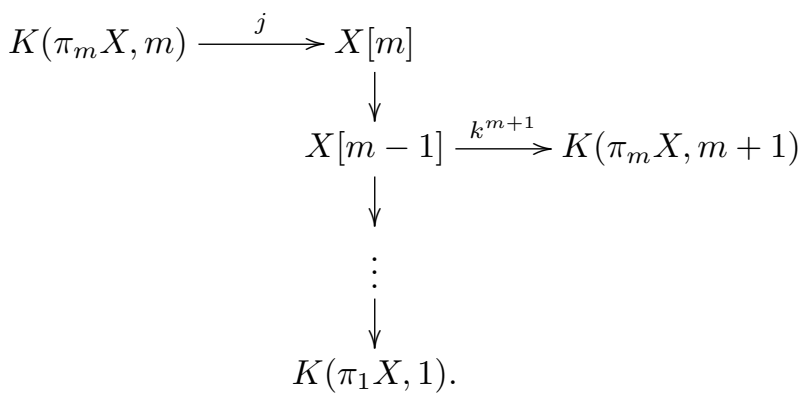

The map $j: K\left(\pi_{m} X, m\right) \rightarrow X[m]$ induces an isomorphism on the $m$-th homotopy groups. Therefore $\Omega^{m-2} j: K\left(\pi_{m} X, 2\right) \rightarrow \Omega^{m-2} X[m]$ and its adjoint map $\Sigma^{m-2} K\left(\pi_{m} X, 2\right) \rightarrow X[m]$, which belongs to $\pi_{m-2} \operatorname{map}_{*}\left(K\left(\pi_{m} X, 2\right), X[m]\right)$, are not nullhomotopic. This contradicts the fact that $\operatorname{map}_{*}\left(K\left(\pi_{m} X, 2\right), X[m]\right)$ is weakly contractible.

\section{References}

1. W. Browder, Torsion in H-spaces, Ann. of Math. 74 (1961), 24-51.

2. H. Cartan, Algèbres d'Eilenberg-MacLane et homotopie, Exposés 2 à 16, Séminaire Henri Cartan, Ecole Normale Supérieure, Paris, 1956.

3. A. Clément, Integral cohomology of finite Postnikov towers, Ph.D. thesis, University of Lausanne, Switzerland, 2002. (See http://www.unil.ch/cyberdocuments/ and http://magma.epfl.ch/aclement)

4. A. ClÉMEnT, The Eilenberg-Mac Lane Machine, a $C++$ program that computes the integral cohomology of Eilenberg-Mac Lane spaces, University of Lausanne, Switzerland, 1998-2002. (See http://www.unil.ch/cyberdocuments/ and http://magma.epfl.ch/aclement)

5. F. R. Cohen And F. P. Peterson, Some free modules over the cohomology of $K(Z / 2 Z, n)$ : a short walk in the Alps, in: Contemporary Mathematics 265 (2000), 7-20.

6. J. LANnes And L. Schwartz, À propos de conjectures de Serre et Sullivan, Invent. Math. 83 (1986), 593-603.

7. R. LEVI, On finite groups and homotopy theory, Memoirs of the A.M.S. 567, vol. 118, 1995.

8. J. MCCleARY, A user's guide to spectral sequences, Cambridge studies in advanced mathematics 58, 2nd edition (Cambridge Univ. Press, 2000).

9. J. W. Milnor And J. C. Moore, On the structure of Hopf algebras, Ann. of Math. 81 (1965), 211-264.

10. J.-P. SERRE, Homologie singulière des espaces fibrés, Ann. of Math. 54 (1951), 425-505.

11. J.-P. Serre, Cohomologie modulo 2 des complexes d'Eilenberg-MacLane, Comment. Math. Helv. 27 (1953), 198-232.

Institute of Mathematics, Faculty of Sciences, University of Lausanne Current address: Alain Clément, Louis-Meyer 9, CH-1800 Vevey, Switzerland E-mail address: clement.alain@mac.com 